\title{
Desempenho anaeróbico lático e aeróbico de mulheres com ciclo menstrual ovulatório
}

\author{
RIBEIRO, Cléo Pereira' ${ }^{1}$ ROTH, Maria Amélia² e ROMBALDI, Airton José ${ }^{3}$
}

\begin{abstract}
Resumo
Este trabalho analisou o comportamento das potências anaeróbica lática e aeróbica durante as fases folicular e lútea do ciclo menstrual. O exercício físico induz respostas semelhantes entre os sexos. Entretanto, a mulher sofre flutuações significativas nos caminhos fisiológicos, uma vez que seus hormônios não são secretados continuamente durante o mês. Esse padrão rítmico é conhecido por ciclo feminino ou menstrual: este apresenta duas fases - folicular e lútea. Alguns autores afirmam que durante a fase lútea o desempenho declina, enquanto outros não encontraram diferenças. A presente amostra foi formada por 5 mulheres, com ciclo ovulatório, idade entre 20 e 40 anos, moderadamente ativas. Para verificar a potência anaeróbica lática, utilizou-se o teste em pista de atletismo de 40 segundos e para verificar a potência aeróbica, foi aplicado um teste progressivo máximo com análise direta do $\mathrm{VO}_{2}$ em ciclo ergômetro. Utilizou-se a estatística descritiva, o teste $t$ e a correlação de Pearson com um nível de significância de $p<0,05$. Não houve diferenças significativas nas potências anaeróbica lática e aeróbica. As variáveis fisiológicas, volume ventilatório e temperatura corporal, apresentaram respostas significativamente diferentes. Constatou-se ainda, casos que apresentaram queda significativa no $\mathrm{VO}_{2}$ máximo.
\end{abstract}

Palavras-Chaves: mulher, atividade física, ciclo menstrual.

\section{Introdução}

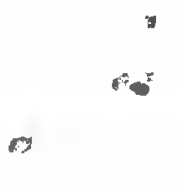

A igualdade de direitos, nos últimos anos, possibilitou à mulher mostrar seu potencial em todas as infinitas situações intelectuais e físicas, deixando de lado muitas crendices populares.

Para Wells (1992), homens e mulheres não são iguais, existem diferenças que vão desde características sexuais à grandezas constitucionais, anatômicas e fisiológicas, sendo estas últimas, focos de grandes estudos.

\footnotetext{
' Prof. Esp. em Ciência do Movimento Humano.

${ }^{2}$ Prof ${ }^{3}$ Assis. Ms. do Centro de Educação Física e Desportos da UFSM.

${ }^{3}$ Prof. Dr. da Escola Superior de Educação Física da Universidade Federal de Pelotas.
}

Revista Kinesis, Santa Maria, $N^{o}$ Especial, p. 130-154, nov. 2001 
Segundo Powers \& Howley (2000); Guyton \& Hall (1998), muitos estudos foram desenvolvidos, visando comparar os sexos perante esforço, as principais conclusões demonstraram que, as respostas fisiológicas femininas são quase idênticas se não iguais as masculinas.

Entretanto, Guyton \& Hall (1998) destacam que, as diferenças hormonais entre homens e mulheres podem determinar mudanças nessas conclusões fisiológicas, explicando, talvez em parte, algumas questões. Embora siga, a exemplo do sistema masculino, uma hierarquia de diferentes hormônios, a mulher não os secreta em quantidades constantes e contínuas como o homem, mas sim, de forma acentuadamente diferente. Seus anos reprodutivos, caracterizam-se por alterações rítmicas mensais na secreção dos hormônios. Esse padrão rítmico chama-se ciclo sexual feminino ou, de forma menos precisa, ciclo menstrual.

As alterações hormonais, de acordo com Speroff, Glass \& Kase (1994), podem ainda, desencadear, outra característica muito marcante na mulher, a "Síndrome de Tensão Pré Menstrual (STPM)", essa, caracteriza-se pelo surgimento cíclico de uma série de sintomas, em grau suficiente para interferir na sua qualidade de vida.

Foram encontrados mais de 150 sintomas, entre físicos e psicológicos, relacionados a STPM, sendo os mais comuns: ansiedade, depressão, irritabilidade, retenção hídrica e aumento de peso, inchaço abdominal, etc (Wells, 1992; Ransom \& Moldenhauer, 1998).

Segundo Guyton \& Hall (1998); Aires (1999); Mellion (1997); Raff, (2000), o ciclo menstrual é dividido em duas fases, ocorrendo entre elas à ovulação, a primeira é chamada de folicular ou proliferativa na qual o hormônio estrogênio predomina e a segunda é conhecida como lútea ou secretora com predomínio do hormônio progesterona. Já para Halbe (1990), o ciclo menstrual divide-se em três fases: folicular, ovulatória e lútea.

Mellion (1997); Powers \& Howley (2000) afirmam que, durante os ciclos menstruais, as concentrações e proporções de estrogênio e progesterona podem alterar a dinâmica do volume vascular, a temperatura, a ventilação e o metabolismo do substrato, todavia, não existem estudos conclusivos que mostrem as variações na performance durante o mesmo.

Fox, Foss \& Keteyian (2000) comentam que, do prisma fisiológico, as respostas metabólicas e cardiovasculares em repouso, exercício submáximo e máximo, não sofreriam mudanças significativas durante as fases do ciclo menstrual.

Num estudo conduzido por Doolittle \& Engebretsen (1972), não foi possível estabelecer relações entre a corrida de 12 minutos, a corrida de 584 metros e a corrida de 1,5 minuto, com as fases do ciclo menstrual.

Stephenson, Kolka \& Wilkerson (1980), também não encontram, em nenhuma intensidade de exercício, diferenças no que se referia ao $\mathrm{VO}_{2}$, limiar anaeróbico, variáveis respiratórias, assim como o tempo de esgotamento, com 6 mulheres que completaram uma série de exercícios até exaustão sobre uma bicicleta ergométrica durante os dias 2, 8, 14, 20, e 26 dos seus ciclos.

Entretanto, Williams \& Krahenbuhl (1997) analisando a economia de corrida, durante cinco fases do ciclo menstrual, de oito mulheres treinadas e exercitadas a 55 e $80 \%$ do $\mathrm{VO}_{2}$ máximo e durante repouso, encontraram resultados que discordam dos anteriores, demonstrando que a taxa de $\mathrm{VO}_{2} \mathrm{em} \mathrm{ml} / \mathrm{kg}$.min e a ventilação em l/min em repouso, eram significativamęnte maiores, durante a fase luteal. Da mesma forma, a velocidade de corrida, a $80 \%$ do consumo de oxigênio, apresentou um maior gasto energético para ser mantida, durante a fase luteal, o que não foi observado, quando a demanda de oxigênio representava $55 \%$ do máximo. 
Dados fornecidos por Allsen, Parsons \& Bryce (1977) concordam com os anteriores, destacando que, o melhor desempenho físico, ocorria entre o período pós-menstrual imediato até o $15^{\circ}$ dia do ciclo menstrual.

Resumindo, vários estudos sobre o rendimento esportivo e as fases do ciclo menstrual, Brooks-gunn, Gargiulo \& Warren (1986) afirmam que, 40 a $60 \%$ das esportistas não demonstraram alterações no desempenho e que, 15 a $30 \%$ obtiveram suas piores respostas ao esforço, durante as fases pré-menstrual, menstrual ou ambas.

Muitas mulheres, nas últimas três décadas, vêm envolvendo-se em programas de atividade física na intenção de melhorar seu estilo de vida e sua saúde. Houve um crescimento explosivo no número de meninas e mulheres envolvidas com jogos esportivos, programas de exercícios e atividades recreativas. A saúde pública nos Estados Unidos informa que hoje $45 \%$ das mulheres, entre 20 e 40 anos, participam de programas de atividade física regular. Isto traduz aproximadamente 8 a 10 milhões de mulheres jovens, representando, assim, um setor significante da população feminina (Costa \& Guthrie, 1994).

Nieman (1999) reforça as colocações anteriores dizendo que, atualmente, as mulheres estão cada vez mais participando de atividades físicas recreativas e competitivas extenuantes, antigamente repudiadas por elas.

O aumento, cada vez mais significativo, dessa população nos programas de exercícios físicos, por si só, justificaria a necessidade de um melhor esclarecimento sobre a relação mulher e atividade física, pois a literatura atual é escassa e controvertida.

Nesse contexto, muita preocupação surgiu entre participantes, treinadores e médicos, relativo aos efeitos do exercício durante o ciclo menstrual. Existe um corpo submetido a exercício e um espectro largo de alterações durante o ciclo menstrual, como: insuficiente fase lútea durante ciclos de duração normal, fases lúteas encurtadas, irregularidade menstrual, anovulação e amenorréia (Costa \& Guthrie, 1994; Mellion, 1997; Fleck \& Kraemer, 1999).

Para Wells (1992) os estudos realizados, até o momento, são polêmicos e deixam margem para muitos questionamentos.

Em 5 anos avaliando e prescrevendo atividade física para o público feminino, tem-se observado que muitas mulheres não apresentam o mesmo desempenho físico perante testes de esforço e/ou atividades aeróbicas, essas flutuações de desempenho se manifestam contrastando com os períodos do ciclo menstrual. Obviamente uma correta prescrição de exercícios parte de um processo de avaliação prévia, essa deve ser padronizada e fidedigna. Será que o critério de fidedignidade entre teste e reteste não é influenciado pelas fases do ciclo menstrual?

Neste sentido, este estudo objetivou determinar quais as respostas das potências anaeróbica lática e aeróbica de mulheres com ciclo ovulatório, durante as fases do ciclo menstrual. Além disto, buscou-se responder:

- verificar nas fases folicular e lútea, a ventilação, a frequiência cardíaca, pressão arterial e temperatura corporal;

- verificar através de questionário, a subjetividade da relação das fases do ciclo com o desempenho físico;

- verificar os níveis séricos hormonais durante a fase lútea do ciclo. 


\section{Metodologia}

\section{Amostra}

Foram sujeitos do estudo 5 mulheres moderadamente ativas, com ciclo ovulatório. A seleção da amostra foi por voluntariedade. Não apresentavam nenhum tipo de doença presente ou problema físico, que interferisse no estudo. Todas apresentavam um ciclo menstrual médio de 28 a 30 dias.

\section{Descrição do estudo}

Este estudo caracterizou-se como descritivo. Foi utilizado um grupo de mulheres que não usavam contraceptivo hormonal oral (Tabela 1).

$\mathrm{GE}=$ grupo do estudo formado por 5 sujeitos.

O grupo foi submetido aos mesmos testes, durante duas fases distintas do ciclo menstrual, a primeira chamada de fase folicular (FF) no $11^{\circ}$ ou $12^{\circ}$ dia e a segunda chamada de fase lútea (FL) no $23^{\circ}$ ou $24^{\circ}$ dia. Foi considerado o primeiro dia de fluxo sangüíneo como o marcador para estabelecer o primeiro dia do ciclo menstrual, assim como as fases para o estudo.

$\mathrm{FF}=$ fase folicular $\left(11^{\circ}\right.$ ao $12^{\circ}$ dia do ciclo menstrual $)$.

$\mathrm{FL}=$ fase lútea $\left(23^{\circ}\right.$ ao $24^{\circ}$ dia do ciclo menstrual).

Tabela 1 - Descrição do estudo

\begin{tabular}{lll}
\hline Grupo & Pré-teste (FF) & Pós-teste (FL) \\
\hline GE & $\mathrm{O} 1$ & $\mathrm{O} 2$ \\
\hline
\end{tabular}

\section{Variáveis do estudo}

\section{a) Fases do ciclo menstrual}

Foi escolhido um ciclo menstrual para análise. Os testes de potência anaeróbica lática e potência aeróbica, foram aplicados em dois períodos distintos do ciclo. Primeiro período, fase folicular, no $11^{\circ}$ ou $12^{\circ}$ dia, segundo período, fase lútea, no $23^{\circ}$ ou $24^{\circ}$ dia, todos contados a partir do primeiro dia de fluxo menstrual.

\section{b) Potência anaeróbica lática}

Método: para determinação da potência anaeróbica łáctica foi utilizado o teste de 40 segundos proposto por Matsudo (1983), em pista de atletismo demarcada metro a metro.

\section{Estimativa da potência anaeróbica:}

Para estimar a potência anaeróbica, foi utilizada a equação proposta por Powers \& Howley (2000):

EQUAÇÃO 1 - Fórmula para estimar a potência anaeróbica

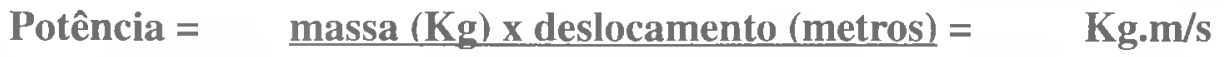
tempo 


\section{c) Potência aeróbica ( $\mathrm{VO}_{2}$ Máximo)}

Método: o protocolo utilizado foi o de Balke, apud Leite (1993), onde a duração de cada estágio é de 2 minuto, a velocidade é constante entorno de 50 a 60 rotações por minuto, com incrementos de $25 \mathrm{~W}$ a cada 2 minutos. Após o teste de potência anaeróbica lática, o sujeito descansou por vinte minutos, sendo os últimos cinco minutos, utilizados para coleta do $\mathrm{VO}_{2} \mathrm{em}$ repouso. $\mathrm{O} \mathrm{VO}_{2}$ foi verificado diretamente durante as fases de repouso, exercício e recuperação e estimado de forma indireta através da seguinte equação:

\section{EQUAÇÃO 2 - Fórmula para estimar a potência aeróbica}

$$
\mathrm{VO}_{2} \mathrm{máx}=\frac{(12.2 \times \text { carga em Watts })+300}{\text { Peso }(\mathrm{kg})}=\quad \mathrm{ml} / \mathrm{kg} \cdot \mathrm{min}
$$

Onde: carga em Watts = carga referente ao último estágio completo.

\section{d) Pressão arterial e temperatura cutânea}

Após a chegada da avaliada ao laboratório (e a permanência sentada por um período não inferior a 15 minutos) foi registrado a temperatura cutânea (medida axilar, por um período de 3 minutos) e a pressão arterial de repouso. Também foi registrado a temperatura cutânea bem como a pressão arterial imediatamente após os 2 minutos de recuperação passiva após o teste ergométrico. A verificação da pressão arterial foi feita no braço direito e a temperatura cutânea na axila esquerda.

\section{e) Sensação subjetiva de interferência no desempenho físico}

A coleta de dados subjetivos se deu através de entrevista, buscando informações através de perguntas pré definidas sobre a interferência das fases do ciclo menstrual no desempenho físico.

\section{f) Alimentação}

As pessoas que participaram da amostra foram orientadas, para que mantivessem seus hábitos alimentares inalterados no tocante ao tipo e a quantidade durante todo o mês destinado ao estudo.

\section{g) Síndrome de tensão pré-menstrual}

Foi utilizado o questionário proposto por Muse, apud Resener et al. (1993), para avaliar os sintomas pré-menstruais, nos dois períodos do ciclo menstrual, folieular e lúteo. O somatório total obtido determinou o grau de presença dos sintomas ng, dia do teste. Não foi realizado nenhum estudo prévio da amostra para confirmar se os sujeitos apresentavam STPM.

\section{h) Estado motivacional para realização dos testes}

As pessoas que participaram da amostra foram motivadas sempre a realizarem o melhor possível durante os testes físicos, buscando atingirem o máximo de suas capacidades.

\section{i) Temperatura ambiente}

A temperatura ambiente foi controlada somente durante o teste de potência aeróbica, esta permaneceu entre 15 e $22^{\circ} \mathrm{C}$ durante as duas fases. Para a realização do teste de potência 
anaeróbica lática, encontrou-se uma temperatura que variou entre 10 e $26^{\circ} \mathrm{C}$ na fase folicular e 9 a $24^{\circ} \mathrm{C}$ na fase lútea.

\section{j) Dosagem hormonal (progesterona)}

Essa coleta aconteceu no $23^{\circ}$ dia contado a partir do $I^{\circ}$ dia de fluxo menstrual. O método utilizado foi quimioluminescência.

Procedimento utilizado:

O Laboratório responsável pela coleta utilizou o Kit Progesterona Immulite fase sólida, estritamente produzido para diagnóstico in vitro, usado para a determinação quantitativa da progesterona em soro. Esse procedimento é um ensaio tipo enzima-imunoensaio quimiluminescente, competitivo, com o ligante marcado.

A coleta foi realizada uma única vez por um funcionário treinado (enfermeira), através de punção venosa em tubos (sem anticoagulante). Retirou-se uma quantidade de sangue entorno de 4 a $10 \mathrm{ml}$, que deveria possibilitar pelo menos $125 \mathrm{~mL}$ de soro, que posteriormente foi utilizado para análise.

O presente estudo considerou como ciclo ovulatório, somente aqueles em que a quantidade de progesterona indicava níveis superiores a $5 \mathrm{ng} / \mathrm{ml}$, conforme proposto por Donadio, Lopes \& Melo (1997).

OBS: O kit utilizado é produzido por: Diagnostic Products Corporation. Representante exclusivo no Brasil, DPC Medlab, PRODUTOS MÉDICO-HOSPITALARES LTDA, São Paulo, SP.

\section{Caracterização da amostra}

Foram utilizados como indicadores do perfil da amostra as seguintes variáveis: idade, estatura, massa corporal total e composição corporal.

A densidade corporal foi encontrada através do somatório de sete dobras cutâneas, tricipital, subescapular, peitoral, axilar, supra-ilíaca, abdominal e coxa medial, desenvolvidas por Jackson, Pollock \& Ward e o \% de gordura estimado pela equação de Siri, apud Pollock \& Wilmore (1993).

$$
\begin{aligned}
& \text { EQUAÇÃO } 3 \text { - Fórmula para verificar a densidade corporal } \\
& \mathrm{Dc}=1,0970-0,00046971(\mathrm{a} 7)+0,00000056\left(a^{7} 7\right)^{2}-0,00012828(\text { ID }) \\
& \text { Onde: } \\
& \text { å } 7 \text { = somatório das sete dobras cutâneas } \\
& \text { ID = Idade do indivíduo }
\end{aligned}
$$

$$
\begin{aligned}
& \text { EQUAÇÃO } 4 \text { - Cálculo do percentual de gordura } \\
& \% \mathrm{G}=(495 / \mathrm{Dc})-450
\end{aligned}
$$

\section{Tratamento estatístico}

Os valores foram expressos como médias $(\mathrm{X}) \pm$ desvios-padrão (DP). Foram utilizados o coeficiente de variação (CV\%), valor mínimo (Mín.) e máximo (Máx.), teste t de student 
para amostras dependentes e correlação produto-momento de Pearson (r), sendo aceito o nível de significância de $\mathrm{p}<0,05$. Foi empregado o pacote Statística para Windows, versão 5.0 da Statsoft, Inc.

\section{Limitações do estudo}

O presente estudo limitou-se a avaliar somente um ciclo menstrual isolado. Em função da disponibilidade pessoal dos sujeitos e dos custos que envolveram o estudo a amostra foi pequena, talvez não seja possível generalizar os resultados.

É possível que o teste de potência anaeróbica lática, tenha interferido nas respostas do teste de potência aeróbica, uma vez que o período de descanso entre eles, foi de apenas 20 minutos.

\section{Resultados e discussão}

Com a intenção de servir como indicadores de tais respostas, as seguintes variáveis fisiológicas foram verificadas: $\mathrm{VO}_{2}$, ventilação pulmonar, freqüência cardíaca, temperatura corporal, pressão arterial sistólica e diastólica.

Ainda, relacionado ao teste de potência aeróbica, foi investigado o comportamento do $\mathrm{VO}_{2}$, da ventilação pulmonar e da freqüência cardíaca em dois diferentes percentuais de esforço, 50 e $85 \%$. Para cada variável fisiológica foi realizado uma média do grupo em dois estágios distintos, as cargas entre 75 e 100 watts foram encontradas para $50 \%$ e as cargas ente 125 e 175 watts para $85 \%$ do máximo atingido. Convêm lombrar que o protocolo de Balke para ciclo ergômetro apresenta incrementos a cada 2 minutos. O valor médio de cada sujeito foi calculado através dos valores do primeiro e segundo minutos, no estágio referente a intensidade desejada.

A verificação através de exame bioquímico, das concentrações de progesterona, serviram como indicador da presença de um ciclo ovulatório bifásico, com duas fases distintas, folicular e lútea.

Qualquer abreviatura utilizada para expor uma variável foi acrescida da sigla FF (fase folicular indicando pré-teste) e FL (fase lútea indicando pós-teste).

Primeiramente, com a finalidade de caracterizar a amostra, ap̉resenta-se a TABELA 02 com as variáveis: idade (ID) estatura (EST), massa corporal total (MCEFF, MCTFL) e percentual de gordura (\%GFF, \%GFL).

Tabela 2 - Médias, desvios - padrão (DP), coeficiente de variação (CV\%), mínimo (Mín.), máximo (Máx.) da amostra.

\begin{tabular}{ccccc}
\hline N $=5$ & Média \pm DP & CV \% & Mín. & Máx. \\
\hline ID (anos) & $31,00 \pm 6,78$ & 21,87 & 20,00 & 38,00 \\
EST (m) & $1,62 \pm 0,08$ & 4,94 & 1,50 & 1,71 \\
MCTFF (Kg) & $57,42 \pm 7,29$ & 12,70 & 47,30 & 67,20 \\
MCTFL (Kg) & $57,94 \pm 7,14$ & 12,32 & 48,25 & 67,75 \\
\%GFF & $20,21 \pm 3,48$ & 17,22 & 16,33 & 24,36 \\
\%GFL & $19,94 \pm 3,89$ & 19,51 & 16,48 & 24,39 \\
\hline
\end{tabular}

Revista Kinesis, Santa Maria, $N^{\circ}$ Especial, p. 136-154, nov. 2001 
Através da Tabela 2 pode-se verificar que o grupo não apresentou diferenças significativas no seu perfil durante o estudo, principalmente na massa corporal total $(p=0,16)$ e a quantidade de gordura $(\mathrm{p}=0,77)$. Portanto sugere-se que não houve interferência dessas variáveis nos resultados obtidos.

O método de dobra cutânea utilizado para estimar a gordura corporal pode implicar em erros grosseiros influenciados por fatores como a habilidade do avaliador, equação utilizada e tipo de compasso (Heyward \& Stolarczyk, 2000). Para amenizar esses problemas, a coleta foi realizada por um único avaliador, a equação para predizer a densidade corporal e o percentual de gordura foram validadas para mulheres numa ampla faixa etária, englobando assim a amostra do presente estudo.

Alguns estudos indicaram que baixos níveis de gordura corporal podem desencadear amenorréia e distúrbios na produção hormonal (Powers \& Howley, 2000; Fox et al., 2000; Weineck, 2000; Raff, 2000; Nieman, 1999), influenciando assim o ciclo menstrual. Um percentual de gordura abaixo de $13 \%$ poderia induzir tais problemas (Katch \& Mcardle, 1996).

Powers \& Howley (2000), dizem que um percentual de gordura entre 15 e $25 \%$ para mulheres, estaria associado a níveis ideais para a saúde e condicionamento físico. A amostra, do presente estudo, encontra-se situado nesta recomendação. O valor médio foi de 20,26 e 19,83\% de gordura corporal entre as fases FF e FL, respectivamente. Este valor não seria considerado um desencadeador de problemas relacionados ao ciclo menstrual.

O segundo objetivo especifico foi determinar as respostas da potência anaeróbica lática. As variáveis de controle utilizadas foram: freqüência cardíaca máxima atingida (FCMFF, FCMFL), distância em metros percorrida (DMPFF, DMPFL) e potência em Kg.m/s (PFF, PFL), conforme Tabela 3.

Tabela 3 - Médias, desvios - padrão (DP), coeficiente de variação (CV\%), mínimo (Min.), máximo (máx.) da potência anaeróbica lática.

\begin{tabular}{|c|c|c|c|c|}
\hline$N=5$ & Média \pm DP & $\mathrm{CV} \%$ & Mín. & Máx. \\
\hline FCMFF & $168,50 \pm 2,97$ & 1,76 & 166 & 173 \\
\hline FCMFL & $172,20 \pm 3,96$ & 2,30 & 167 & 178 \\
\hline DMPFF (m) & $199,00 \pm 21,08$ & 10,59 & 163 & 217 \\
\hline DMPFL (m) & $198,60 \pm 21,50$ & 10,83 & 167 & 219 \\
\hline PFF (Kg.m/s) & $286,52 \pm 47,59$ & 16,61 & 232,28 & 336.00 \\
\hline PFL (Kg.m/s) & $288,01 \pm 43,28$ & 15,03 & 243,82 & 335,36 \\
\hline
\end{tabular}

Os resultados encontrados sobre a potência anaeróbica lática.jnão apresentaram diferenças estatísticas entre as médias apresentadas, freqüência cardíaca máxima $(\mathrm{p}=0,17)$, distância percorrida $(\mathrm{p}=0,92)$ e potência anaeróbica expressa em $\mathrm{Kg} \cdot \mathrm{m} / \mathrm{s}(\mathrm{p}=0,82)$. Observando a freqüência cardíaca máxima atingida no final dos 40 segundos pode-se inferir que os sujeitos empenharam-se durante as duas fases do ciclo.

Os fatores climáticos como velocidade do vento $(\mathrm{V}, \mathrm{p}=0,45)$ e temperatura ambiente $(\mathrm{T}$ $\left.{ }^{\circ} \mathrm{C}, \mathrm{p}=0,56\right)$, não apresentaram variações estatisticamente significativas, sugerindo pouca ou nenhuma interferência nos resultados, como é possível observar na Tabela 4. 
Tabela 4 - Médias, desvios - padrão (DP), coeficiente de variação (CV\%), mínimo (mín.), máximo (máx.) das variáveis ambientais da potência anaeróbica lática.

\begin{tabular}{ccccc}
\hline $\mathrm{N}=5$ & Média $\pm \mathrm{PD}$ & CV \% & Mín. & Máx. \\
\hline $\mathrm{V} \mathrm{FF}(\mathrm{Km} / \mathrm{h})$ & $10,60 \pm 10,29$ & 97,04 & 2 & 25 \\
$\mathrm{~V} \mathrm{FL}(\mathrm{Km} / \mathrm{h})$ & $7,20 \pm 2,17$ & 30,11 & 5 & 10 \\
$\mathrm{~T}^{\circ} \mathrm{C} \mathrm{FF}$ & $18,80 \pm 6,69$ & 45,17 & 10 & 26 \\
$\mathrm{~T}^{\circ} \mathrm{C} \mathrm{FL}$ & $18,40 \pm 7,30$ & 39,68 & 9 & 24 \\
\hline
\end{tabular}

As condições de atrito (piso) da pista foram semelhantes nas duas fases.

Os resultados encontrados por este estudo, corroboram com Doolittle \& Engebretsen (1972); Fox et al. (2000) e Giacomoni et al. (2000), uma vez que, não foi possível estabelecer relação estatística significativa entre a potência anaeróbica lática e as fases do ciclo menstrual.

Os resultados permitem dizer que, a aplicação de testes de cunho anaeróbico lático, não sofreria interferência do ciclo menstrual, pois as mudanças ocorridas durante o ciclo feminino, não interferiram na rota metabólica anaeróbica, pelo menos na quantidade de trabalho possível de ser executado. Entretanto o custo energético para a realização desse volume de trabalho poderia, ser diferente. De acordo Maughan, Gleeson \& Greenhaff (2000), esse tipo de exercício depende exclusivamente das concentrações de glicose circulante e glicogênio estocado na musculatura, sendo assim, qualquer alteração na disponibilidade desses substratos poderia redirecionar a rota metabólica, caso a intensidade diminui-se, ou ainda, provocar a parada imediata do trabalho.

A quantidade e a velocidade de quebra desses substratos, poderia ser traduzida através das concentrações de lactato. No entanto, esse importante marcador do metabolismo anaeróbico não foi investigado, dificultando assim uma maior confirmação da conclusão obtida neste estudo, o que não invalida o resultado encontrado.

Outras variáveis, como o ritmo de corrida, imposto pelo próprio sujeito e o fato desses não estarem adaptados a exigências de esforço máximo, talvez tenham interferido nos resultados.

Seguindo os objetivos propostos, buscou-se verificar a potência aeróbica através da medida direta e indireta do consumo de $\mathrm{O}_{2}$. As variáveis encontradas nas Tabelas 5, 6, 7 e 8, demonstram os resultados.

Tabela 5 - Médias, desvios - padrão (DP), coeficiente de variação (CV\%), mínimo (mín.), máximo (máx.), de medida direta do $\mathrm{VO}_{2}$ em repouso, máximo e potência máxima atingida.

\begin{tabular}{ccccc}
\hline $\mathrm{N}=5$ & Média $\pm \mathrm{DP}$ & $\mathrm{CV} \%$ & Mín. & Máx. \\
\hline $\mathrm{VO}_{2} \mathrm{R}$ FF (ml/kg.min) & $4,10 \pm 1,19$ & $29,06 \Theta_{\bullet}$ & 2,10 & 5,10 \\
$\mathrm{VO}_{2} \mathrm{R}$ FL (mi/kg.min) & $4,70 \pm 0,58$ & 12,31 & 4,00 & 5,50 \\
$\mathrm{VO}_{2}$ Máx FF (ml/kg.min) & $37,58 \pm 1,81$ & $\approx_{4,83}$ & 34,80 & 39,40 \\
$\mathrm{VO}_{2}$ Máx FL (ml/kg.min) & $35,96 \pm 6,39$ & 17,77 & 27,10 & 43,50 \\
$\mathrm{VO}_{2}$ Máx FF (L/min) & $2,17 \pm 0,30$ & 13,95 & 1,87 & 2,62 \\
$\mathrm{VO}_{2}$ Máx FL (L/min) & $2,09 \pm 0,43$ & 20,52 & 1,58 & 2,66 \\
Watts FF & $170,00 \pm 27,39$ & 16,11 & 150 & 200 \\
Watts FL & $165,00 \pm 33,54$ & 20,33 & 125 & 200 \\
\hline
\end{tabular}

Os resultados demonstram não existir diferenças estatisticamente significativas em nenhuma das variáveis apresentadas, concordando com os resultados apresentados por Wells, \& Horvath (1974); Doolittle \& Engebretsen (1972); Stephenson et al. (1980) e (1982); Fox et al. (2000); Eston \& Burke, apud Wells (1992); Desouza et al. (1990) e Lebrun et al. (1995). Entretanto 
autores como Williams \& Krahenbuhl (1997); Hessemer \& Buck (1985); Allsen et al. (1977), demonstram que o ciclo menstrual pode interferir nas repostas cardiorrespiratórias.

Observando $\mathrm{O} \mathrm{VO}_{2}$ em repouso, constata-se que durante a fase lútea os valores encontrados foram maiores, porém não houve diferença estatística $\left(\mathrm{VO}_{2}\right.$ repouso $\left.\mathrm{p}=0,35\right)$. Isso talvez se deva ao tamanho da amostra utilizada ou ainda o que é mais provável, pela necessidade de ressintetizar as concentrações de fosfato de creatina armazenada nos músculos, bem como remover o lactado produzido pelo teste de 40 segundos. É possível que as concentrações de lactato e conseqüentemente de $\mathrm{H}^{+}$, durante a fase lútea tenham sido maiores do que na fase folicular. Sabe-se que um aumento no consumo de oxigênio poderia acelerar a ressíntese do lactato e remoção dos $\mathrm{H}^{+}$, explicando assim as maiores taxas de oxigênio encontradas durante a fase lútea.

Durante esforço submáximo, 50 e $85 \%$ da carga máxima atingida, o consumo de oxigênio manteve-se estável durante o ciclo menstrual. Não houve alteração significativa no volume de oxigênio utilizado para manter a mesma carga de esforço em nenhuma das fases ( $\mathrm{VO}_{2}$ a $50 \% \mathrm{ml} /$ $\left.\mathrm{kg} \cdot \min \mathrm{p}=0,86 \mathrm{e} \mathrm{L} / \mathrm{min} \mathrm{p}=1,00 ; \mathrm{VO}_{2} 85 \% \mathrm{ml} / \mathrm{kg} \cdot \min \mathrm{p}=0,39 \mathrm{e} \mathrm{L} / \mathrm{min} \mathrm{p}=0,47\right)$. Isso demonstra que a intensidade de treinamento prescrita, moderada ou intensa, não exige um aporte calórico diferenciado durante o ciclo menstrual para ser mantida, Tabela 6.

Tabela 6 - Médias, desvios - padrão (DP), coeficiente de variação (CV\%), mínimo (mín.), máximo (máx.), do volume de oxigênio a 50 e $85 \%$ da carga máxima atingida.

\begin{tabular}{ccccc}
\hline $\mathrm{N}=5$ & Média $\pm \mathrm{DP}$ & $\mathrm{CV} \%$ & Mín. & Máx. \\
\hline $\mathrm{VO}_{2} 50 \% \mathrm{FF}(\mathrm{ml} / \mathrm{kg} \cdot \mathrm{min})$ & $18,49 \pm 3,01$ & 16,28 & 14,05 & 22,55 \\
$\mathrm{VO}_{2} 50 \% \mathrm{FL}(\mathrm{ml} / \mathrm{kg} \cdot \mathrm{min})$ & $18,24 \pm 2,54$ & 13,93 & 15,30 & 20,95 \\
$\mathrm{VO}_{2} 85 \% \mathrm{FF}$ (ml/kg.min) & $29,22 \pm 3,18$ & 10,88 & 24,50 & 32,15 \\
$\mathrm{VO}_{2} 85 \% \mathrm{FL}$ (ml/kg.min) & $28,34 \pm 3,59$ & 12,67 & 23,60 & 32,10 \\
$\mathrm{VO}_{2} 50 \% \mathrm{FF}(\mathrm{L} / \mathrm{min})$ & $1,05 \pm 0,19$ & 18,10 & 0,75 & 1,25 \\
$\mathrm{VO}_{2} 50 \% \mathrm{FL}(\mathrm{L} / \mathrm{min})$ & $1,05 \pm 0,15$ & 14,29 & 0,89 & 1,27 \\
$\mathrm{VO}_{2} 85 \% \mathrm{FF}(\mathrm{L} / \mathrm{min})$ & $1,69 \pm 0,30$ & 17,75 & 1,35 & 2,07 \\
$\mathrm{VO}_{2} 85 \% \mathrm{FL}(\mathrm{L} / \mathrm{min})$ & $1,65 \pm 0,30$ & 18,18 & 1,38 & 1,99 \\
\hline
\end{tabular}

Entretanto, os resultado do presente estudo não estão de acordo com as evidências apresentadas por Williams \& Krahenbuhl (1997). Esses autores afirmam que intensidades elevadas, porém submáximas, determinam um comportamento diferenciado do consumo de oxigênio durante as fases do ciclo menstrual, sendo maior durante a fase lútea e menor na folicular, para uma mesma velocidade de corrida.

Certamente o principal fator causador dessa divergência entrề os resultados obtidos por Williams \& Krahenbuhl (1997), e os detectados por este estudo, «reside no tipo de ergômetro utilizado. Na bicicleta ergométrica não existe a necessidade de suportar o peso corporal, o que já acontece na esteira rolante. A massa muscular envolvida para pedalar não é a mesma para correr, isso certamente provoca um consumo de oxigênio diferenciado. Ainda a eficiência mecânica produzida por cada atividade, pedalar ou correr, não é a mesma, dificultando comparações.

Sobre o $\mathrm{VO}_{2}$ máximo medido diretamente, encontrou-se valores ligeiramente inferiores durante a fase lútea $\left(\mathrm{VO}_{2}\right.$ máximo $\left.\mathrm{ml} / \mathrm{kg} \cdot \min \mathrm{p}=0,48 \mathrm{e} \mathrm{L} / \mathrm{min} \mathrm{p}=0,52\right)$ no entanto, a carga máxima atingida não apresentou variação durante o estudo (Watts $p=0,37$ ), (Tabela 5).

A comparação entre os valores obtidos de $\mathrm{VO}_{2}$ máximo através de medida direta e indireta, demonstraram diferenças estatisticamente significativas. Ao comparar as formas de medida durante a fase folicular do ciclo constata-se um maior valor estimado em contraste com um 
menor verificado ( $\mathrm{VO}_{2}$ máximo em $\mathrm{ml} / \mathrm{kg} \cdot \mathrm{min}, \mathrm{p}=0,02 \mathrm{e} \mathrm{L} / \mathrm{min}, \mathrm{p}=0,03$ ), da mesma forma aconteceu durante a fase lútea ( $\mathrm{VO}_{2}$ máximo em $\left.\mathrm{ml} / \mathrm{kg} \cdot \min \mathrm{p}=0,04 \mathrm{e} \mathrm{L} / \mathrm{min}, \mathrm{p}=0,04\right)$.

O valor verificado ou medido, refere-se a análise das concentrações dos gases exalados durante o teste. Esse procedimento é reconhecido como o de maior precisão na literatura. Entretanto, é um meio oneroso para ser utilizado para verificação de massa. Para resolver esse problema, muitas equações foram validadas buscando estimar indiretamente o $\mathrm{VO}_{2}$ máximo. Essa estimativa baseia-se principalmente na sobrecarga imposta e algumas variáveis fisiológicas. A principal meta em comparar os dois procedimentos, reside na margem de erro existente entre eles, somada a uma possível interferência do ciclo feminino.

Através dos resultados podemos constatar uma superestimação dos valores encontrados através da estimativa indireta. Isto se deve provavelmente ao tipo de equação utilizada para estimar o $\mathrm{VO}_{2}$ máximo. É possível que esta não apresente uma correlação adequada com o aparelho de medida direta utilizado neste estudo, ocasionando assim, tais diferenças, conforme Tabela 7 .

As fases do ciclo parecem não interferir nos valores encontrados indiretamente (fases FF $\mathrm{x} \mathrm{FL}$, medida indireta $\mathrm{ml} / \mathrm{kg} \cdot \mathrm{min}, \mathrm{p}=0,85$ ), assim como foi constatado através da medida direta do consumo máximo de oxigênio.

Tabela 7 - Médias, desvios - padrão (DP), coeficiente de variação (CV\%), mínimo (mín.), máximo (máx.), do $\mathrm{VO}_{2}$ máximo direto e indireto.

\begin{tabular}{llrrr}
\hline \multicolumn{1}{c}{$\mathrm{N}=5$} & Média $\pm \mathrm{DP}$ & $\mathrm{CV} \%$ & Mín. & Máx. \\
\hline $\mathrm{VO}_{2}$ Direto FF $(\mathrm{ml} / \mathrm{kg} \cdot \mathrm{min})$ & $37,58 \pm 1,81 *$ & 4,82 & 34,80 & 39,40 \\
$\mathrm{VO}_{2}$ Indireto FF (ml/kg.min) & $41,28 \pm 3,45$ & 8,36 & 37,37 & 45,03 \\
\hline $\mathrm{VO}_{2}$ Direto FL (ml/kg.min) & $35,96 \pm 6,39 *$ & 17,77 & 27,10 & 43,50 \\
$\mathrm{VO}_{2}$ Indireto FL (ml/kg.min) & $39.85 \pm 5.43$ & 13.63 & 31.25 & 44.73 \\
\hline $\mathrm{VO}_{2}$ Direto FF (L/min) & $2,17 \pm 0,30 *$ & 13,82 & 1,87 & 2,62 \\
$\mathrm{VO}_{2}$ Indireto FF (L/min) & $2,37+0,33$ & 13.92 & 2.13 & 2.74 \\
$\mathrm{VO}_{2}$ Direto FL (L/min) & $2,09 \pm 0,43 *$ & 20,57 & 1,58 & 2,66 \\
\hline
\end{tabular}

* diferença estatisticamente significante $\mathrm{p}<0,05$.

Curiosamente, dois sujeitos da amostra apresentaram um declínjo no $\mathrm{VO}_{2}$ máximo durante a segunda fase, esses sujeitos apresentavam as maiores concentrações de progesterona encontradas no grupo (valores superiores a $20 \mathrm{ng} / \mathrm{ml}$ ). Assim buscou-se correlacionar os valores de progesterona com o consumo máximo de oxigênio (Tabela 8), a fim de busçăr algumas explicações.

Tabela 8 - Coeficiente de correlação entre potência aeróbica (medida direta) e níveis de progesterona.

\begin{tabular}{cc}
\hline Variáveis $\mathrm{N}=5$ & Progesterona $(\mathrm{ng} / \mathrm{ml}) \mathrm{r}$ \\
\hline $\mathrm{VO}_{2} \mathrm{R} \mathrm{FF}(\mathrm{ml} / \mathrm{kg} \cdot \mathrm{min})$ & $-0,66$ \\
$\mathrm{VO}_{2} \mathrm{R} \mathrm{FL} \mathrm{(ml/kg.min)}$ & $-0,54$ \\
$\mathrm{VO}_{2} \mathrm{Máx} F \mathrm{FF}(\mathrm{ml} / \mathrm{kg} \cdot \mathrm{min})$ & $-0,95^{*}$ \\
$\mathrm{VO}_{2} \mathrm{Máx} \mathrm{FL}(\mathrm{ml} / \mathrm{kg} \cdot \mathrm{min})$ & $-0,96^{*}$ \\
$\mathrm{VO}_{2} \mathrm{Máx} \mathrm{FF}(\mathrm{L} / \mathrm{min})$ & $-0,45$ \\
$\mathrm{VO}_{2}$ Máx FL $(\mathrm{L} / \mathrm{min})$ & $-0,89 *$ \\
\hline
\end{tabular}

* correlação é significante $\mathrm{p}<0,05$.

Revista Kinesis, Santa Maria, $N^{o}$ Especial, p. 140-154, nov. 2001 
A análise estatística indica uma forte correlação entre as concentrações de progesterona e as respostas obtidas no $\mathrm{VO}_{2}$ máximo durante a fase folicular e lútea. Sugere-se, que grandes concentrações de progesterona provocariam um declínio no consumo máximo de oxigênio o que conseqüientemente prejudicaria o andamento de atividades que dependem do sistema aeróbico para promover energia. Essa sugestão, certamente precisa ser melhor investigada, pois a amostra utilizada foi pequena, o que coloca sob suspeita tal inferência.

Entretanto, se essa inferência for verdadeira, o seguinte exemplo poderia acontecer: uma mulher foi avaliada durante a fase folicular, o $\mathrm{VO}_{2}$ máximo encontrado foi $45 \mathrm{ml} / \mathrm{kg}$. min. Ao se prescrever uma intensidade de $80 \%$ encontraríamos $36 \mathrm{ml} / \mathrm{kg}$. min. Entretanto durante a fase lútea seu $\mathrm{VO}_{2}$ máximo caiu para $40 \mathrm{ml} / \mathrm{kg}$.min, pois as maiores concentrações de progesterona durante essa fase provocaram uma menor capacidade máxima de oxigênio. A diferença entre os valores máximos é de $5 \mathrm{ml} / \mathrm{kg}$.min, o que certamente provocaria um aumento da intensidade durante a segunda fase do ciclo. Os resultados se inverteriam, caso o teste tivesse sido feito na fase lútea. Segundo o ACMS (1996), uma unidade metabólica em repouso é igual a 3,5 ml/ kg.min que é igual a $1 \mathrm{Km} / \mathrm{h}$. Uma alteração desse porte, seria significante para aumentar ou diminuir uma determinada intensidade de esforço.

O exemplo acima faz refletir, pois o profissional menos informado poderia incorrer num erro de $1 \mathrm{Km} / \mathrm{h}$ para mais ou menos que certamente provocaria um comprometimento da atividade prescrita. Este problema pode ser ainda maior se somarmos a margem de erro encontrada em função do tipo de ergômetro e equação utilizada para estimar o $\mathrm{VO}_{2}$ máximo.

Pode-se ainda inferir que as pequenas diferenças encontradas no $\mathrm{VO}_{2}$ máximo, são decorrentes da adaptação dos sujeitos com os procedimentos do teste. Os que tiveram dificuldade na primeira testagem poderiam falsear os resultados da segunda, não se empenhando e conseqüentemente não atingindo a potência máxima.

Outra variável observada durante o teste de potência aeróbica foi a ventilação pulmonar em litros por minuto, em repouso (VER), exercício submáximo, 50 e $85 \%$ da carga máxima atingida (VE 50 e 85\%) e exercício máximo (VEM), conforme Tabela 9 e 10.

Tabela 9 - Médias, desvios - padrão (DP), coeficiente de variação (CV\%), mínimo (mín.), máximo (máx.), do volume ventilatório, repouso e máximo.

\begin{tabular}{ccccc}
\hline N $=5$ & Média \pm DP & CV \% & Mín. & Máx. \\
\hline VER FF (L/min) & $8,24 \pm 2,75$ & 33,35 & 5,60 & 12,50 \\
VER FL (L/min) & $9,66 \pm 1,80$ & 18,64 & 7,40 & 11,90 \\
VEM FF (L/min) & $79,82 \pm 13,97$ & 17,51 & 58,10 & 92,50 \\
VEM FL (L/min) & $75,36 \pm 17,52$ & 23,25 & 58,40 & 98,30 \\
\hline
\end{tabular}

O presente estudo demonstrou que a ventilação pulmonar durante o repouso $(\mathrm{p}=0,07)$ e exercício máximo $(\mathrm{p}=0,55)$, não foram estatisticamente diferentes, entretanto houve uma forte tendência durante repouso, o que concordaria com os estudos de Williams \& Krahenbuhl (1997), que também encontraram durante o repouso, porem não em esforço, valores de ventilação/min, significativamente maiores durante a fase lútea.

É possível que o aumento no volume ventilatório em repouso, tenha acontecido pelo mesmo fator observado com o $\mathrm{VO}_{2}$ em repouso, ou seja, maiores concentrações de lactato provocadas pelo teste de 40 segundos realizado na segunda fase do ciclo menstrual. Powers \& Howley (2000), dizem que um aumento de $\mathrm{H}^{+}$e $\mathrm{PCO}_{2}$ no sangue estimularia os quimiorreceptores 
aórticos a aumentar o volume ventilatório. Sendo assim o aumento em repouso, seria uma conseqüência do teste de 40 segundos e não uma interferência do ciclo menstrual.

Uma maior temperatura corporal, um aumento nos níveis de potássio e catecolaminas no sangue, são considerados fatores secundários que poderiam contribuir para o controle da ventilação, pelo menos durante o exercício intenso (Powers \& Howley, 2000).

Sendo assim o aumento da temperatura corporal encontrado na fase lútea, poderia desencadear um maior volume ventilatório em repouso.

No que se refere à intensidade do exercício, 50 e $85 \%$, não foi possível estabelecer diferença estatística (VE 50\% p =0,63 e VE $85 \%$ p =0,57). Sem dúvida os mesmos fatores responsáveis pelos resultados observados no consumo de oxigênio, provavelmente fizeram-se presentes na capacidade ventilatória, Tabela 10.

Tabela 10 - Médias, desvios - padrão (DP), coeficiente de variação (CV\%), mínimo (mín.), máximo (máx.), do volume ventilatório a 50 e $85 \%$ da carga máxima atingida.

\begin{tabular}{ccccc}
\hline $\mathrm{N}=5$ & Média $\pm \mathrm{DP}$ & $\mathrm{CV} \%$ & Mín. & Máx. \\
\hline VE 50\% FF (L/min) & $31,61 \pm 5,66$ & 17,91 & 24,75 & 39,30 \\
VE 50\% FL (L/min) & $32,36 \pm 3,74$ & 11,56 & 28,50 & 37,10 \\
VE 85\% FF (L/min) & $55,08 \pm 11,60$ & 23,10 & 44,20 & 69,20 \\
VE 85\% FL (L/min) & $54,20 \pm 9,94$ & 19,06 & 44,45 & 65,65 \\
\hline
\end{tabular}

Ainda, a capacidade ventilatória foi correlacionada com os níveis de progesterona, pois foi documentado por Bonekat et al. (1987); Schoene et al. (1981), que durante a fase lútea ocorreria aumentos significativos na capacidade ventilatória de mulheres eumenorréicas com altas concentrações de progesterona. A literatura tem demonstrado que o hormônio progesterona é um potente estimulador do centro respiratório.

Os resultados encontrados corroboram com as afirmações anteriores, pois encontrou-se correlação entre as concentrações de progesterona e a ventilação em repouso durante a fase lútea e uma forte tendência também na fase folicular, já em esforço máximo não foi estabelecido nenhuma correlação, no entanto houve uma forte tendência entre a ventilação máxima durante a fase lútea.

O fato do presente estudo não ter encontrado correlação entre as taxas de progesterona com o volume ventilatório máximo, pode estar relacionado a alguns fatores, entre eles, a quantidade de sujeitos que compuseram a amostra e os níveis individuais de progesterona produzidas após a ovulação.

Uma pequena amostra certamente dificulta qualquer tentativa de correlação entre os dados e o fato da maioria dos sujeitos da amostra não apresentar granđ̆es concentrações de progesterona sérica, certamente poderiam influenciar os resultados obtidos. A Tabela 11, demonstra a correlação entre a ventilação e os níveis de progesterona.

Tabela 11 - Coeficiente de correlação entre o volume ventilatório e os níveis de progesterona.

\begin{tabular}{cc}
\hline Variáveis N=5 & Progesterona $(\mathrm{ng} / \mathrm{ml}) \mathbf{r}$ \\
\hline VER FF (L/min) & $-0,81$ \\
VE R FL (L/min) & $-0,96^{*}$ \\
VE M FF (L/min) & $-0,03$ \\
VEM FL (L/min) & $-0,82$ \\
\hline
\end{tabular}

* correlação é significante $\mathrm{p}<0,05$.

Revista Kinesis, Santa Maria, $N^{o}$ Especial, p. 142-154, nov. 2001 
Os valores de freqüência cardíaca observados no teste de potência aeróbica, não demonstraram diferenças estatisticamente significativas durante repouso $(p=0,80)$ e máxima atingida $(p=0,73)$, concordando com os estudos de Eston \& Burke apud Wells (1992).

As fases do ciclo menstrual parecem não interferir nas respostas da freqüência cardíaca. É possível constatar que os valores em repouso demonstram indivíduos com um grau moderado de condição física, também é possível inferir que as variáveis que provocam alterações nessa variável fisiológica mantiveram-se constantes durante as fases folicular e lútea. Analisando a freqüência cardíaca máxima atingida durante o teste, pode-se verificar que o valor atingido, é muito próximo do estimado através da fórmula (220 - a idade), tomando o valor médio da idade do grupo, chega-se a um valor de $189 \mathrm{bpm}$, esse fica ligeiramente acima do valor atingido em teste, demonstrando que os indivíduos empenharam-se igualmente durante teste e reteste. A Tabela 12, demonstra os valores em repouso (FCR) e máximos atingidos (FCM).

Tabela 12 - Médias, desvios - padrão (DP), coeficiente de variação (CV\%), mínimo (mín.), máximo (máx.), da frequiência cardíaca de repouso e máxima atingida em teste.

\begin{tabular}{ccccc}
\hline $\mathrm{N}=5$ & Média $\pm \mathrm{DP}$ & CV \% & Mín. & Máx. \\
\hline FCR FF bpm & $66,00 \pm 5,79$ & 8,77 & 58 & 71 \\
FCR FL bpm & $66,80 \pm 11,21$ & 16,78 & 53 & 79 \\
FCM FF bpm & $184,40 \pm 5,55$ & 3,01 & 180 & 194 \\
FCM FL bpm & $183,40 \pm 8,32$ & 4,54 & 173 & 196 \\
\hline
\end{tabular}

Também foi investigada a frequiência cardíaca nas intensidades submáximas de 50 e $85 \%$ (Tabela 13). Os resultados indicam que independentemente, da intensidade prescrita, o comportamento cardíaco mostrou-se muito semelhante durante as fases folicular e lútea (FC 50\%, $p=0,97$ e $85 \%, p=0,84)$, demonstrando pouca ou nenhuma interferência do ciclo menstrual nas respostas cardíacas.

Muitos fatores foram relacionados pela literatura como elementos influenciadores do ritmo cardíaco, entre eles os estímulos psicológicos e ambientais (ACMS, 1996), os grupos musculares exercitados, o tipo de exercício, contínuo ou intervalado, a ingestão alimentar, o estado de hidratação e a idade (Mcardle et al., 1998), são considerados os principais. Até o momento, não foram evidenciadas relações entre a freqüuência cardíaca e as fases do ciclo menstrual (Eston \& Burke, apud Wells, 1992).

Tabela 13 - Médias, desvios - padrão (DP), coeficiente de variação (CV\%), mínimo (mín.), máximo (máx.), da frequiência cardíaca a 50 e $85 \%$ da carga máxima atingida em teste.

\begin{tabular}{|c|c|c|c|c|}
\hline $\mathbf{N}=\mathbf{5}$ & Média \pm DP & $\begin{array}{c}\mathrm{CV} \\
\%\end{array}$ & Mín. & Máx. \\
\hline FC 50\% FF bpm & $147,60 \pm 6,26$ & 4,24 & 138,00 & 153,50 \\
\hline FC 50\% FL bpm & $147,50 \pm 10,19$ & 6,91 & 129,50 & 154,00 \\
\hline FC $85 \%$ FF bpm & $172,90 \pm 5,52$ & 3,19 & 166,50 & $\mathbf{1 8 1 , 5 0}$ \\
\hline FC $85 \%$ FL bpm & $173,30 \pm 8,50$ & 4,90 & 160,00 & 183,50 \\
\hline
\end{tabular}

Observando a pressão arterial em repouso, constata-se que esta apresentou um comportamento semelhante à freqüência cardíaca, mantendo-se constante durante as duas fases do ciclo menstrual. Os valores encontrados referentes ao grau de significância foram os seguintes: pressão sistólica, $p=0,62$ e diastólica, $p=0,18$. Não foi estabelecida nenhuma diferença estatística significativa entre a pressão arterial em repouso durante as fases do ciclo avaliadas. A TABELA 14 , demonstra os valores de pressão arterial durante o repouso. As variáveis apresentadas são: sistólica (S) e diastólica (D). 
Tabela 14 - Médias, desvios - padrão (DP), coeficiente de variação (CV\%), mínimo (mín.), máximo (máx.), da pressão arterial de repouso.

\begin{tabular}{lcccc}
\hline N = 5 & Média \pm DP & CV \% & Mín. & Máx. \\
\hline S FF & $112,00 \pm 8,37$ & 7,47 & 100 & 120 \\
S FL & $110,00 \pm 7,07$ & 6,43 & 100 & 120 \\
D FF & $74,00 \pm 8,94$ & 12,09 & 60 & 80 \\
D FL & $70,00 \pm 7,07$ & 10,10 & 60 & 80 \\
\hline
\end{tabular}

Novamente pode-se verificar que as respostas pressóricas encontradas na fase de recuperação, mostraram-se muito semelhantes ao comportamento da freqüência cardíaca e pressão arterial em repouso, não sendo possível estabelecer nenhuma evidência estatisticamente significante durante as fases do ciclo menstrual (sistólica, $\mathrm{p}=0,83$ e diastólica, $\mathrm{p}=0,70$ ), conforme Tabela 15 .

Resumindo: as variáveis fisiológicas, freqüência cardíaca e pressão arterial em repouso encontradas no presente estudo, não foram influenciadas pelo ciclo reprodutivo feminino.

Tabela 15 - Médias, desvios - padrão (DP), coeficiente de variação (CV\%), mínimo (mín.), máximo (máx.), da pressão arterial após esforço, recuperação.

\begin{tabular}{|c|c|c|c|c|}
\hline $\mathrm{N}=5$ & Média \pm DP & $\mathrm{CV} \%$ & Mín. & Máx. \\
\hline S FF & $114,00 \pm 11,40$ & 10,00 & 100 & 130 \\
\hline$S \mathrm{FL}$ & $112,00 \pm 13,04$ & 11,64 & 100 & 130 \\
\hline D FF & $74,00 \pm 8,94$ & 12,09 & 70 & 90 \\
\hline D FL & $72,00 \pm 4,47$ & 6,21 & 70 & 80 \\
\hline
\end{tabular}

A investigação da temperatura cutânea veio demonstrar uma diferença estatisticamente significativa em repouso $(p=0,03)$, conforme Tabela 16 . Vale lembrar que a temperatura corporal central é maior que a verificada na superfície cutânea. De acordo com os valores estabelecidos por esse estudo é possível sugerir, que durante a fase lútea ocorreu um aumento significativo da temperatura corporal em repouso.

Tabela 16 - Médias, desvios - padrão (DP), coeficiente de variação (CV\%), mínimo (mín.), máximo (máx.), da temperatura cutânea em repouso.

\begin{tabular}{lcccc}
\hline $\mathrm{N}=5$ & Média $\pm \mathrm{DP}$ & $\mathrm{CV} \%$ & Mín. & Máx. \\
\hline $\mathrm{T}^{\circ} \mathrm{C}$ FF & $36,14 \pm 0,53 *$ & 1,47 & 35,60 & 36,90 \\
$\mathrm{~T}^{\circ} \mathrm{C}$ FL & $36,40 \pm 0,65$ & 1,79 & 35,60 & 37,20 \\
\hline
\end{tabular}

* diferença estatisticamente significante $\mathrm{p}<0,05$.

Isso demonstra que os mecanismos termorregulatórios encontram-se acelerados durante a fase lútea. Conforme afirmam Powers \& Howley (2000); Weineck, (2000); Wells, (1992); Aires (1999), a temperatura corporal apresenta uma relação muito intima com as fases do ciclo menstrual, elevando-se depois da ovulação e permanece ligeiramente alta ao longo da fase lútea, acontecendo em repouso como também em exercícios máximơe submáximo. Para Halbe (1990), essas variações que a temperatura basal apresenta ao longo do ciclo estão condicionadas pela ação termogênica da progesterona ao nível do centro termorregulador hipotalâmico. Na fase folicular a temperatura em geral é inferior a $36,5^{\circ} \mathrm{C}$, apresentando queda próximo à ovulação.

Gonzalez \& Blanchard (1998), também encontraram relação entre a temperatura corporal e o ciclo menstrual de seis mulheres com idade entre 18 e 29 anos, não aclimatadas, em repouso, durante as fases folicular ( $2^{\circ}$ ao $6^{\circ}$ dia) e luteal ( $19^{\circ}$ ao $23^{\circ}$ dia), onde foi constatado vasoconstrição periférica intensa na fase folicular e grande produção de calor durante a fase lútea, quando submetidas ao frio. Embora os autores não tenham relatado nenhuma evidência sobre a produção de calor em exercício, Williams \& Krahenbuhl (1997), afirmam que a mesma velocidade de 
corrida exigiu uma maior quantidade de oxigênio durante a fase lútea, provocando assim uma maior produção de calor.

Contrariando os resultados anteriores Wells \& Horvath (1974), não encontraram nenhuma diferença entre mulheres destreinadas, porém aclimatadas, na temperatura cutânea durante as diferentes fases do ciclo.

A temperatura corporal tem sido utilizada para indicar a presença de ovulação, entretanto, esses aumentos podem ser conseqüências de outras causas, o que certamente falsearia o real estado do ciclo. É provável que os estudos que não encontraram relação dessa com as fases menstruais, tenham especulado somente a temperatura corporal, o que não garante $100 \%$ de certeza, a presença de um ciclo bifásico com ovulação. Este problema metodológico, certamente é o principal observado entre os estudos.

A temperatura cutânea após recuperação demonstrou estatisticamente uma diferença significativa $(\mathrm{p}=0,05)$ durante a fase lútea, Tabela 17. O valor encontrado foi menor que o verificado durante a fase folicular. Nenhum estudo relatou valores de temperatura corporal após exercício.

Tabela 17 - Médias, desvios - padrão (DP), coeficiente de variação (CV\%), mínimo (mín.), máximo (máx.), da temperatura cutânea após exercício, recuperação.

\begin{tabular}{ccccc}
\hline $\mathrm{N}=5$ & Média $\pm \mathrm{DP}$ & $\mathrm{CV} \%$ & Mín. & Máx. \\
\hline $\mathrm{T}^{\circ} \mathrm{C}$ FF & $36,34 \pm 0,53^{*}$ & 1,46 & 35,70 & 37,10 \\
$\mathrm{~T}^{\circ} \mathrm{C}$ FL & $35,64 \pm 0,56$ & 1,57 & 35,00 & 36,90 \\
\hline
\end{tabular}

* diferença estatisticamente significante $\mathrm{p}<0,05$.

É possível que esse fenômeno tenha ocorrido em função de uma maior redistribuição sangüínea para a superfície cutânea conforme afirmam Powers \& Howley (2000); Fox et al. (2000). Mesmo que exista uma maior produção de calor durante a fase lútea (Gonzalez \& Blanchard, 1998; Williams \& Krahenbuh1, 1997), os mecanismos termorregulatórios conseguiram agir de forma mais eficiente nesta fase quando comparada a folicular, facilitando assim um rápido resfriamento após exercício.

Outro objetivo específico estabelecido neste estudo, foi a avaliação subjetiva através de questões pré-elaboradas, relacionando a interferência das fases do ciclo menstrual no desempenho físico.

Os valores encontrados demonstraram que a maioria das entrevistadas, $60 \%$ ( 3 sujeitos) alegavam perceber diferença no seu desempenho físico durante o ciclo menstrual e identificam a fase pré-menstrual e menstrual como a de pior desempenho. 40\% (2 sujeitos) dizem que não percebem essa relação. Embora a maioria da amostra tenha indiçad̆o interferência subjetiva no desempenho, isso não afetou as respostas durante os testes, pois não houve redução nas potências anaeróbica lática e aeróbica durante as fases do ciclo, Figura 1.

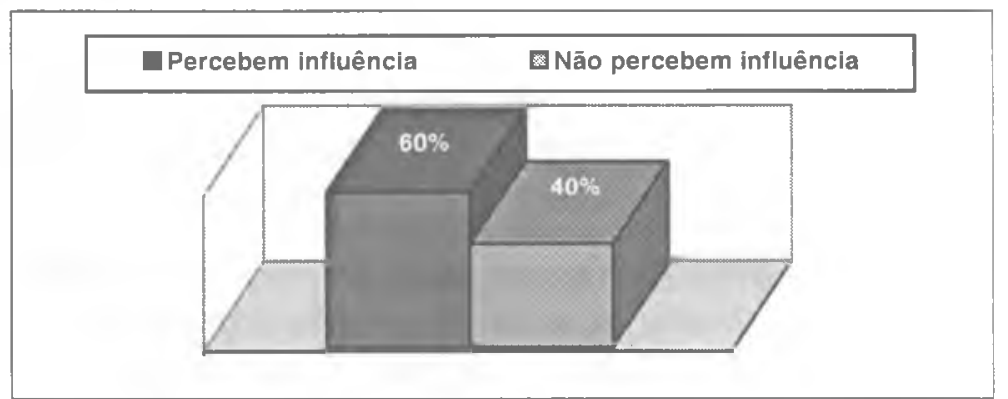

Figura 1 - Avaliação subjetiva da influência do ciclo menstrual no desempenho físico 
As concentrações de progesterona foram analisadas através do método chamado quimioluminescência com a finalidade de detectar a presença de ciclo ovulatório.

A Figura 2, demonstra os valores individuais de progesterona de cada componente do grupo utilizado na amostra.

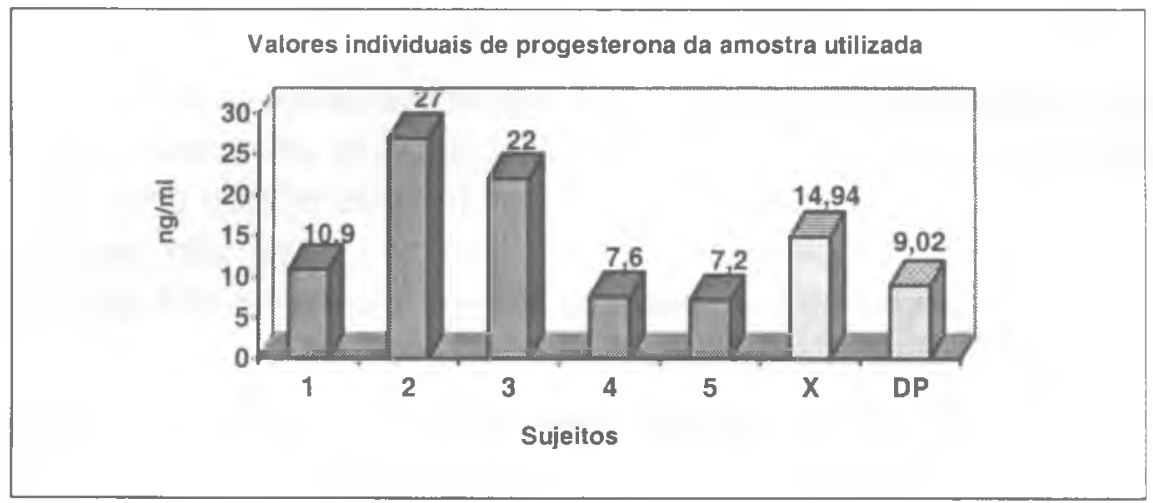

Figura 2 - Valores individuais de progesterona

Com o objetivo de avaliar a interferência da STPM, foi utilizado nesse estudo um questionário para avaliar o grau de presença dos sintomas. Não existiu diferença estatisticamente significativa, entre as duas fases do ciclo no grupo testado $(p=0,10)$, conforme demonstrado na Tabela 18. Entretanto, pode-se detectar já na fase folicular, a presença de alguns sintomas. Estes, costumam aparecer nos últimos 7 a 10 dias do ciclo menstrual (Speroff et al., 1994), desaparecendo logo após a menstruação (Reid \& Yan, 1981). Sendo assim, é possível, sugerir que esta variável não interferiu nas respostas da potência anaeróbica lática e aeróbica.

Tabela 18 - Médias, desvios - padrão (DP), coeficiente de variação (CV\%), mínimo (mín.), máximo (máx.), dos sintomas de tensão pré-menstrual (somatório geral).

\begin{tabular}{ccccc}
\hline $\mathrm{N}=5$ & Média $\pm \mathrm{DP}$ & $\mathrm{CV} \%$ & Mín. & $\begin{array}{c}\text { Má } \\
\mathrm{x} .\end{array}$ \\
\hline FF & & & 0 & 6 \\
FL & $2,60 \pm 2,61$ & 100,30 & 0 & 21 \\
\hline
\end{tabular}

* diferença estatisticamente significante $\mathrm{p}<0,05$.

Foram encontrados mais de 150 sintomas, entre físicos e psicológicos, relacionados à STPM, sendo os mais comuns: ansiedade, depressão, irritabilidade, dor de cabeça, tensão mamaria, retenção hídrica, inchaço abdominal, edema periférico, aumento de apetite, tran'stornos gastrointestinais, vertigens, mudanças no humor, etc., não restringindo apenas a estes (Wells, 1992; Ransom \& Moldenhauer, 1998). A quantidade exata, no entanto é difícil de ser determinada por causa da variabilidade dos sintomas e pela dificuldade de serem quantificados (Speroff et al., 1994).

Neste estudo, somente um sujeito classificou como severo a presença de alguns sintomas. De acordo com os critérios estabelecidos por Muse, apud Resener et al. (1993), três sujeitos apresentavam sinais da STPM, o que não interferiu nas repostas da potência anaeróbica lática e aeróbica, pois não houve diferenças nestas, durante o ciclo menstrual.

Comparando as duas fases, pode-se observar uma grande heterogeneidade, 100,30 e $81,85 \%$, FF e FL, respectivamente, de variação ao redor da média. Constata-se ainda, um maior $\mathrm{CV}$ na fase folicular. Pelos resultados infere-se o seguinte comentário: apesar dos estudos demonstrarem que a STPM se faz presente principalmente na segunda fase do ciclo menstrual,

Revista Kinesis, Santa Maria, $N^{o}$ Especial, p. 146-154, nov. 2001 
é possível constatar a existência de alguns sintomas, já na fase folicular. Será que esses realmente existiram ou são reflexos condicionados? Speroff et al. (1994) sugerem que as mulheres foram criadas dentro dessa expectativa de que durante a fase pré-menstrual, vários sintomas como retenção hídrica, dor e distúrbios emocionais, se manifestariam. Ao utilizar estudos retrospectivos, estes sintomas relatados passam a fazer parte deste estereotipo.

Conforme os autores acima, pode-se observar através da história, evidências de tabus relacionados à menstruação, que sem dúvida prejudicam os estudos científicos sobre o assunto.

\section{Conclusões}

No presente estudo ao analisar-se as variáveis propostas, com base na amostra, concluise que não existe diferença nas respostas da potência anaeróbica lática e aeróbica durante ciclos menstruais ovulatórios, em mulheres com idade entre 20 e 40 anos, moderadamente ativas.

Independentemente da intensidade do esforço, as respostas fisiológicas do $\mathrm{VO}_{2}$, da frequiência cardíaca, do volume ventilatório máximo e da pressão arterial, da amostra do estudo, não sofreram interferências das fases do ciclo menstrual.

A temperatura cutânea e o volume ventilatório em repouso foram as variáveis que se mostraram influenciadas durando o ciclo menstrual, demonstrando que existe uma modificação no comportamento de atuação destes mecanismos fisiológicos.

Se estimar indiretamente o consumo máximo de oxigênio, em ciclo ergômetro, pode-se cometer uma superestimativa do real valor de $\mathrm{VO}_{2}$ máximo.

$\mathrm{O}$ estudo encontrou ainda, casos com grandes variações nas respostas de $\mathrm{VO}_{2}$ máximo, durante as fases do ciclo menstrual. Tal fato é de extrema relevância na medida em que reforça a necessidade de uma avaliação individual e mais, que algumas mulheres necessitam ser avaliadas nas duas fases de seu ciclo feminino, a fim de que se possa detectar as flutuações de desempenho.

\section{Referências bibliográficas}

AIRES, M., M., Fisiologia. Rio de Janeiro: Guanabara Koogan, 1999.

ALLSEN, P., E.; PARSONS, P.; BRYCE, G. R.., Effect of menstrual cycle on maximum oxygen uptake. In: Physician and Sports Medicine. Vol.05, $\mathrm{p}_{b}$ 53-55, 1977.

AMERICAN COLLEGE OF SPORTS MEDICINE, - Prova de Esforço e Prescrição de Exercício. Rio de Janeiro: Revinter,1996.

BONEKAT, H., W.; DOMBOVY, M., L.; WILliAMS, T., J.; STAATS, B., A., Progesterone induced changes in exercise performance and ventilatory response. In Medicine \& Science in Sports \& Exercise. Vol.19, p. 118-123, 1987.

BROOKS-GUNN, J.; GARGIULO, J., M.; WARREN, M., P., The effect of cycle phase on the performance of adolescent swimmers. In Physiology Sports Medicine. Vol.14, p. 182, 1986.

COSTA, D., M.; GUTHRIE, S., R., Women and Sport: interdisciplinary perspectives. Califórnia, USA: Human Kinetics. 1994. 
DESOUZA, M., J.; MAGUIRE, M., S.; RUBIN, K.; MARESH, C., M., Effects of menstrual phase and amenorrehea on exercise responses in runners. In Medicine \& Science in Sports \& Exercise. Vol.22, p. 575-580, 1990.

DONADIO, N.; LOPES, J., R., C.; MELO, N., R., Reprodução Humana II, Infertilidade, Anticoncepção, Reprodução Assistida. São Paulo: Organon, 1997.

DOOLITTLE, R., L.; ENGEBRETSEN, J., Performance variations during the menstrual cycle. In Journal of Sports Medicine and Physical Fitness. Vol.12, p. 54-58, 1972.

FLECK, S., J.; KRAEMER, W., J., Fundamentos do Treinamento de Força Muscular. Porto Alegre, RS: Artes médicas, 1999.

FOX, E., L.; FOSS, M., L.; KETEYIAN, S.,J., Bases fisiológicas do exercício e do esporte. Rio de Janeiro: Guanabara Koogan, 2000.

GIACOMONI, M.; BERNARD, T.; GAVARRY, O.; ALTARE, S.; FALGAIRETTE, G., Influence of the menstrual cycle phase and menstrual symptoms on maximal anaerobic performance. In Medicine \& Science in Sports \& Exercise. Vol.32, n.2, p. 486-492, 2000.

GONZALEZ, R., R.; BLANCHARD, L., A.; Thermoregulatory responses to cold transients: effects of menstrual cycle in resting women. In: Journal of Applied Physiology. Vol.85, p. 543-553, 1998.

GUYTON, A., C.; HALL, J., E., Fisiologia humana e mecanismos das doenças. Rio de Janeiro: Guanabara koogan, 1998.

HALBE, H., W., Tratado de Ginecologia; 2a reimpressão, Vol. 1; São Paulo: Roca, 1990.

HESSEMER, V.; BRUCK, K., Influence of menstrual cycle on thermoregulatory, metabolic, and heart rate responses to exercise at night. In: Journal of Applied Physiology. Vol.59, p. 1911-1917, 1985.

HEYWARD, V., H.; STOLARCZYK, L., M., Avaliação da composição corporal aplicada. São Paulo: Manole, 2000.

KATCH, F., I.; McARDLE, W., D., Nutrição, Exercício e Saúde. Rio de Janeiro: Medsi, 1996.

LEBRUN, C., M.; McKENZIE, D., C.; PRIOR, J., C.; TAUNTON, J., E., Effects of menstrual cycle phase on athetic performance. In: Medicine \& Science in Sports \& Exercise. Vol. 27, p. 437-444, 1995.

LEITE, P., F., Fisiologia do exercício, ergometria e condicionamento físico. São Paulo: Robe, 1993.

MATSUDO, V., K., Testes em ciências do esporte. São Caetano do Sul, SP: Ltda, 1983.

MAUGHAN, R.; GLEESON, M.; GREENHAFF, P., L., Bioqû́f́mica do exercício e do treinamento. São Paulo: Manole.

McARDLE, W., D.; KATCH, F., I., \& KATCH V., L., Fisiologia do exercício, energia, nutrição e desempenho humano. 4. ed. Rio de Janeiro: Guanabara Koogan, 1998.

MELLION, M., B., Segredos em Medicina Desportiva. Porto Alegre, RS.: Artes Médicas, 1997.

NIEMAN, D., C., Exercício e Saúde. São Paulo, SP: Manole, 1999.

POLLOCK, M., L., \& WILMORE, J., H., Exercícios na saúde e na doença. São Paulo, SP.: Medsi, 1993.

POWERS, S., K., \& HOWLEY, E., T., Fisiologia do Exercício. São Paulo: Manole, 2000.

RAFF, H., Segredos em fisiologia. Porto Alegre, RS: Artes médicas, 2000.

Revista Kinesis, Santa Maria, $N^{\circ}$ Especial, p. 148-154, nov. 2001 
RANSOM, S., \& MOLDENHAUER, J., Premenstrual syndrome: systematic diagnosis and individualized therapy. The Physician And Sports Medicine. v 26, n 4, April, 1998.

REID, R., L., \& YEN, S., S., C., Premenstrual syndrome. American Journal of Obstetrics Gynecology. v. 139, p. 85-104, 1981.

RESENER, E., V.; SANTOS, J., E.; FERRIANI, R., A.; MOURA, M., D.; SILVA DE SÁ, M., F., Uso de questionário para diagnóstico da síndrome pré-menstrual. Femina. v. 21, p. 1072 1082, 1993.

SCHOENE, R., B.; ROBERTSON, H., T.; PIERSON, D., J., \& PETERSON, A., P., Respiratory drives and exercises in menstrual cycle of athletic and nonathletic women. Journal of Applied Physiology. v. 50, p. 1300-1305, 1981.

SPEROFF, L.; GLASS, R., H., \& KASE, N., G., Clinical Gynecologic Endocrinology and Infertility. Baltimore, Maryland 21202, USA.: Williams \& Wilkins, 1994.

STEPHENSON, L., A.; KOLKA M., A., \& WILKERSON, J., E., Anaerobic threshold, work capacity, and perceived exertion during the menstrual cycle. Medicine \& Science in Sports \& Exercise. v. 12(2), p. 87, (abstract), 1980.

. Metabolic and thermoregulatory response to exercise during the human menstrual cycle. Medicine \& Science in Sports \& Exercise. v. 14, p. 270-275, 1988.

WEINECK, J., Biologia do esporte. São Paulo, SP.: Manole, 2000.

WELLS, C., L., \& HORVATH, S., M., Responses to exercises in a hot environment as related to the menstrual cycle. Journal of Applied Physiology. v. 36, p. 299, 1974.

WELLS, C., L., Mujeres, deporte y rendimiento. Barcelona, España: Paidotribo, Ia ed., 1992.

WILLIAMS, T., J., \& KRAHENBUHL, G., S., Menstrual cycle phase and running economy. Medicine \& Science in Sports \& Exercise. v. 29, n. 12, p. 1609-1618, 1997.

WOLINSKY, I., \& HICKSON, J., F., Nutrição no exercício e no esporte. São Paulo, SP: Roca Ltda, 1996.

\title{
Lactic anaerobic and aerobic performance of women with ovulatory menstrual cycles
}

\begin{abstract}
This study analyzed the effects of menstrual cycle phases on lactic anaerobic anderobic power. Physical exercise induces similar results in both, man and woman. However, a woman suffers significant flotation on the physiologic ways, since hormone secretion is not regular within a period. That rhythmic pattern is known by feminine or menstrual cycle, which presents two phases: follicular and luteal. Some authors state that during the luteal phase the performance declines, while others were unable to find differences. Five women with ovulation cycle, ages between 20 and 40 years old and moderately actives, composed the sample of this study. To verify the lactic anaerobic power, a test in athletics track of 40 seconds was used, and to verify the aerobic power, an incremental exercise test was applied with direct analysis of the $\mathrm{VO}_{2}$ in ergometric cycle. Descriptive statistics, the "t" test and the Pearson correlation, were used with a level of significance of $p<0,05$. There weren't significant differences in lactic anaerobic and aerobic power. Physiologic variables, such as ventilation volume and body temperature, presented significantly,different answers. It was still verified, cases with significant reduction on the maximum $\mathrm{VO}_{2}$.
\end{abstract}

Keywords: woman, physical activity, cycle menstrual. 